\title{
Evidence of a double-double morphology in B 0818+214
}

\author{
A. Marecki and M. Szablewski
}

\author{
Toruń Centre for Astronomy, N. Copernicus University, 87-100 Toruń, Poland \\ e-mail: amr@astro.uni.torun.pl
}

Received 24 July 2009 / Accepted 19 September 2009

\begin{abstract}
The so-called double-double structure in radio sources is the most conspicuous signature of their restarted activity. Observations indicate that in the majority of double-double radio sources (DDRS), the span of the radio lobes is larger than $0.7 \mathrm{Mpc}$. This lower limit is also suggested by theory. However, it seemed likely that the apparent core of B $0818+214$, a radio galaxy with an overall linear size of its radio structure below that limit, could harbour a compact double well aligned with the outer lobes so that the whole object would fulfil the criteria of a DDRS. Here, we present evidence that the central component of B $0818+214$, when magnified through the EVN+MERLIN 18-cm observations, shows two FR II-like lobes. As the separation of the inner lobes is not greater than $5.7 \mathrm{kpc}$, they are immersed in the ISM of the host galaxy. This circumstance is the likely reason why the inner double has become visible, despite the predictions of the theory according to which B $0818+214$ as a whole is too small for a new double to develop inside the cocoon inflated during the previous active phase. Moreover, we speculate that its host galaxy is not active at the moment and so the inner double may be in the coasting phase often observed in other medium-sized symmetric objects with intermittent activity. It could be, therefore, that two different mechanisms of accretion disk instabilities, ionisation and radiation-pressure driven, may be independently responsible for triggering active phases, manifesting as the outer and the inner doubles, respectively.
\end{abstract}

Key words. radio continuum: galaxies - galaxies: active - galaxies: individual: B 0818+214

\section{Introduction}

According to a well-established paradigm, the activity of galaxies can be recurrent. For radio-loud active galaxies, Best (2007) suggested that their activity must be constantly re-triggered. Given that the lobes that build up during the active phase are huge reservoirs of energy - the minimum energy stored in the lobes is in the range of $10^{60}$ to $10^{64} \mathrm{erg}$ (see e.g. Richstone et al. 1998) - it can take up to $10^{8}$ years for them to disappear once the energy supply from the active nucleus cuts off (Komissarov \& Gubanov 1994; Slee et al. 2001). Because the lifetimes of relic lobes are that long, it is plausible that a new active phase of a galaxy may begin before the old lobes have faded out completely, so that its radio images show components resulting from both current and past active periods. The signature of such a renewed activity is most convincing if a large, double-lobed relic structure straddles a pair of young lobes giving rise to the so-called double-double radio source (DDRS) (Lara et al. 1999; Schoenmakers et al. 2000a,b; Saripalli et al. 2002, 2003; Saikia et al. 2006) or even triple double (Brocksopp et al. 2007). DDRSs are almost entirely identified with galaxies; an exception - quasar J0935+0204 (4C 02.27) - has been found by Jamrozy et al. (2009).

Typically, the separation of the outer lobes in DDRSs is not greater than one order of magnitude that of the inner lobes. For example, the outer/inner double size ratio for the sources shown in the seminal paper on DDRSs by Schoenmakers et al. (2000a) ranges from $2($ B $1240+389)$ to 9 (B 1450+333). At first sight, this appears puzzling since the new lobes/hotspots started to expand much later than the old ones, so that they should be much closer to one another. However, hotspots pushed by restarted jets propagate much faster because of the low density of plasma inside the cocoon inflated during the previous phase of activity, compared to those of the old jets moving through the much denser intergalactic medium (IGM) (Kaiser et al. 2000, hereafter $\mathrm{KSR}$ ). Therefore, even if the length of the previous active phase had been the maximum possible $\left(10^{8} \mathrm{yr}\right)$ and assuming that the activity then ceased for a substantial amount of time (also of the order of $10^{8} \mathrm{yr}$ ), once it was re-triggered, the inner structure developed quickly enough so that it is able to catch up with the outer one. It is speculated - see e.g. KSR - that the inner lobes can reach the old cocoon boundary, cross it, and then overtake the outer ones. Consequently, these objects could, paradoxically, also be treated as DDRSs, or "inverted DDRS". PKS 0349-27 seems to be an extreme case of such a scenario - see the image presented by Morganti et al. (1993). Here, the new lobes have overtaken the old ones and appear as completely separate entities. As a result, the whole source is clearly double-double but, unlike in standard DDRSs, in PKS 0349-27, the relics are not the outer but the inner pair.

Because of that rapid development of the inner lobes, it would appear that DDRSs where the separation of the inner lobes is significantly smaller than that of the outer lobes should be rare. Yet, 3C 236 (Schilizzi et al. 2001) and J1247+6723 (Marecki et al. 2003) are such special cases of double-double morphology where the inner part is too compact to be properly imaged in the maps encompassing the outer one and so, as a whole, the source does not appear as a DDRS but as a coredominated triple (CDT), where the alleged core is actually a compact, luminous double source. J1247+6723 is the most extreme case of such situation known to date; here, the ratio of the size of the outer to inner double is five orders of magnitude. 
These two examples are clear indication that at least some CDTs could be concealed DDRSs. Konar et al. (2004) pointed out that the central component of a CDT was more likely to be a compact double if that component had a steep or gigahertz-peaked spectrum (GPS $)^{1}$. Hence, investigations of CDTs with GPS or steep-spectrum cores could lead to discoveries of more DDRSs where a new active phase was triggered only recently so that the linear sizes of the inner doubles are still small.

The catalogue of radio sources extracted from the Faint Images of Radio Sky at Twenty centimetres (FIRST) survey (White et al. 1997) ${ }^{2}$ is very well suited for machine-aided search for CDTs. To this end, Marecki et al. (2006) - hereafter Paper I developed an automated procedure. They also used the GB6 catalogue (Becker et al. 1991) to calculate spectral indices between the frequencies of these two surveys and selected only steepspectrum sources, i.e. those where $\alpha>0.5\left(S \propto v^{-\alpha}\right)$. Obviously, calculation of the spectral indices of the "cores" alone was not possible because of the resolution limit of the GB6 survey so this criterion was fulfilled only approximately. A large fraction of core-jet sources, which typically have flat spectra, possibly were eliminated in this way.

A sample of 15 sources resulted from this selection procedure and is given in Table 1 of Paper I. Their cores were observed with MERLIN at $6 \mathrm{~cm}-$ see Paper I. As a result, B 0818+214 emerged as the most likely candidate for a DDRS since it appeared to have two components in the centre very well aligned with the outer lobes (Paper I, Fig. 1). Admittedly, the inner components were largely asymmetric so, at that stage, a core-jet morphology could not be ruled out. Whether the inner double was actually two FR II-like (Fanaroff \& Riley 1974) lobes could only be decided by means of a low-frequency, high-resolution observation. Thus, B $0818+214$ was followed up at $18 \mathrm{~cm}(1658 \mathrm{MHz})$ with the European VLBI Network (EVN) supplemented with MERLIN. Here, the outcome of these latest observations is reported.

\section{Observations and results}

The overall structure of B $0818+214$ as seen in FIRST image is shown in Fig. 1 of Paper I. The source has three aligned components, the middle one being roughly an order of magnitude stronger than the remaining two - see Table 2 of Paper I. The dominance of the central component is particularly obvious when the peak flux densities are compared: 6-7 mJy for each of the outer lobes and $142 \mathrm{mJy}$ for the "core". The peak flux densities of the lobes are about 3 times less than their total flux densities, which may suggest that these structures are diffuse as expected for relic lobes.

High-resolution observations of the "core" of B $0818+214$ were carried out on 10 June 2007 at $1658 \mathrm{MHz}$ with phase referencing. The network consisted of 14 telescopes of the EVN and MERLIN including the largest two, Lovell and Effelsberg, although due to technical limitations of the combined EVN+MERLIN network, visibilities for only 41 baselines were obtained. Nevertheless, this and the length of the total time spent on the program source - nearly 4 hours divided into 23 10-min. scans spread over $7.5 \mathrm{~h} \mathrm{~s}$ - resulted in a very good, uniform $u$ $v$ coverage and good sensitivity: the noise in the resulting image was $\sim 40 \mu \mathrm{Jy} /$ beam.

The reduction of the data using AIPS was carried out in the standard way and produced the image shown in Fig. 1.

\footnotetext{
${ }_{1}$ In fact, the inner structure of J1247+6723 is a GPS source.

${ }^{2}$ Official website: http://sundog.stsci.edu
}

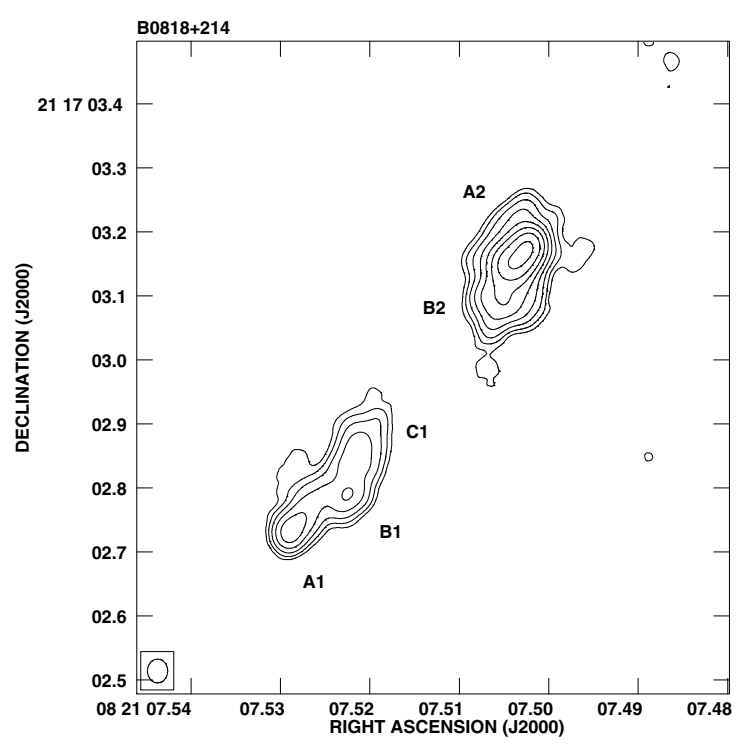

Fig. 1. EVN+MERLIN image of the inner double of B $0818+214$ at $1658 \mathrm{MHz}$. Contours increase by a factor of 2 ; the first contour level corresponds to $\approx 5 \sigma$ level which is $0.21 \mathrm{mJy} / \mathrm{beam}$. The peak flux density is $35.1 \mathrm{mJy} / \mathrm{beam}$. The beam size is $37 \times 32$ milliarcseconds at the position angle of $0^{\circ}$.

Table 1. Flux densities and spectral indices of the components of the inner double.

\begin{tabular}{crrr}
\hline \hline Component & \multicolumn{2}{c}{ Flux density [mJy] } & Spectral index \\
& $1658 \mathrm{MHz}$ & $4994 \mathrm{MHz}$ & \\
\hline $\mathrm{A} 1$ & 11.7 & 6.2 & 0.57 \\
$\mathrm{~B} 1$ & 9.9 & 4.4 & 0.75 \\
$\mathrm{C} 1$ & 10.9 & 2.3 & 1.40 \\
$\mathrm{~A} 2+\mathrm{B} 2$ & 108.6 & 39.1 & 0.93 \\
\hline
\end{tabular}

The 18-cm flux densities of the components of the southeastern lobe (A1, B1, and C1) were measured by fitting Gaussian models using the AIPS task JMFIT and are listed in Col. 2 of Table 1. Column 3 gives the flux densities of their equivalents at $6 \mathrm{~cm}(4994 \mathrm{MHz})$ extracted from the MERLIN image shown in Fig. 1 of Paper I. Due to the limited resolution of the MERLIN 6-cm map, it was not possible to fit a Gaussian model for component B2 of the northwestern lobe in that image. Thus, we used the AIPS TVSTAT utility to measure the total flux densities of that lobe for both $18-\mathrm{cm}$ and $6-\mathrm{cm}$ images. They are given in the bottom row of Table 1.

Based on the flux density figures in Cols. 2 and 3, the spectral indices of the source's inner structure have been calculated and are shown in Col. 4. The values of the indices make it clear that A1, having the flattest spectrum, must be a hotspot. Since the spectrum becomes steeper to the north-west, indicating increased spectral age in that direction, the whole southeastern feature of the inner structure appears as a typical FR II lobe. The morphology and steep spectrum of the A2-B2 region also leaves no doubt that it is the second lobe.

\section{Discussion}

KSR developed a general theory of DDRSs. The main conclusion of their work is that the interior of the old cocoon is an environment where the lobes resulting from a new phase of the 
nuclear activity could not develop easily. For example, KSR show that the scenario of a mere replacement of the cocoon material by the surrounding IGM is inefficient as it would require $10^{8} \mathrm{yr}$ to refill the cocoon once the bow shock has disappeared. Since the above replacement time is close to the decay time of the lobes and disappearance of the bow shock can also take a considerable amount of additional time, DDRSs should rarely be observed as even the largest/oldest ones would never be mature enough (in terms of the internal density) to host a new double.

In an alternative model, KSR posit the presence of warm clouds of gas embedded in the hot IGM. Unlike in the previous scenario, such clouds are able to pass the cocoon border even if there is still a bow shock surrounding the outer source structure. Having done so, they break up into smaller fragments inside the cocoon. This dispersion process takes $\sim 5 \times 10^{7} \mathrm{yr}$. Such a timescale, albeit of comparable magnitude, gives the chance for new lobes and hotspots to develop but only in radio objects with linear sizes of more than $\approx 700 \mathrm{kpc}$. It follows that, even if the activity in a younger source could actually stop and then restart, such an event would remain unobservable.

B 0818+214 lies within the Sloan Digital Sky Survey footprint and it is listed there as an $m_{\mathrm{R}}=21.73$ galaxy. Since no redshift is available, only the upper limits to the linear sizes of both the outer and the inner doubles can be established. Given that for the up-to-date cosmological parameters $\left(H_{0}=71 \mathrm{~km} \mathrm{~s}^{-1} / \mathrm{Mpc}\right.$, $\Omega_{\mathrm{M}}=0.27, \Omega_{\Lambda}=0.73$, Spergel et al. 2003) the maximum possible angular-size distance $D_{\text {Amax }}=1765.1 \mathrm{Mpc}$ (for $z=1.64$ ), the largest ratio of linear to angular sizes for any object in the universe is $8.558 \mathrm{kpc} / /^{\prime \prime}$. Hence, the overall angular size of B $0818+214$, 68", yields its maximum linear size of $582 \mathrm{kpc}$

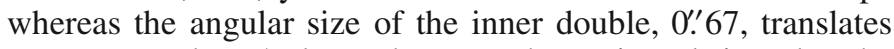
to not more than $5.7 \mathrm{kpc}$. These are the projected sizes, but the absolute sizes are not likely to be significantly greater. This is because if the angle $\theta$ between the source axis and the line of sight was considerably less than $90^{\circ}$ then the light travel-time difference between the epochs in which we observe the outer lobes of $1.9 / \tan \theta$ Myr would be of the order of $10^{6} \mathrm{yr}$. Such a large time lag would inevitably result in a noticeable asymmetry of the outer lobes seen at two well-separated stages of the decay. This is not observed for the outer lobes of B $0818+214$ : their flux densities, armlength ratios, and shapes are very similar.

As the upper limit for the linear sizes of B $0818+214$ is below the threshold required by KSR, it might appear that their model is not applicable to radio sources like B $0818+214$. However, the problem can be resolved taking into account that B $0818+214$ is not a typical DDRS since the inner lobes are two orders of magnitude more compact than the outer ones. Hence, although the inner double is not as small as that of J1247+6723 where we observe an FR II-like double with the linear size of the order of $10 \mathrm{pc}$ (Marecki et al. 2003), here, we have a kpcscale, i.e. medium-sized symmetric object (MSO, Fanti et al. 1995). Because of its size, it is not immersed in the low-density medium of the outer cocoon as in standard DDRSs but rather lies in the ISM of the host galaxy and strongly interacts with it. Such interactions are observed in many compact sources - see Labiano et al. (2005, 2006), Holt et al. (2006), Labiano (2008) - and should be expected in B $0818+214$. This circumstance explains why it could have developed at all and become observable despite the predictions of KSR.

Another potential problem is that the inner lobes of B $0818+214$ are highly asymmetric in terms of their flux densities but, again, this is quite normal as, due to interactions with asymmetric medium in the central regions of their host galaxies, compact steep spectrum (CSS) sources are more asymmetric than larger sources of similar powers (Jeyakumar et al. 2005). The majority of MSOs are CSSs and this is the case for the inner part of B $0818+214$. Another factor that may contribute to its observed asymmetry is the orientation. If the inner lobes have just entered the coasting phase - see the next paragraph - the fast decay of such compact lobes combined with the light travel-time effect could be responsible for the differences in their appearances.

There is yet another characteristic that makes B $0818+214$ a special object. The majority of well-defined DDRSs, apart from two pairs of lobes, have a core. While this is the case for B $0925+420$, B 1240+389, B 1450+333 (Schoenmakers et al. 2000a), B 1834+620 (Schoenmakers et al. 2000b), J0116-473 (Saripalli et al. 2002), and B 1545-321 (Saripalli et al. 2003), we have found no core in B $0818+214$. This could mean that the energy transport from the core has ceased, although, taking into account that at least one hotspot is still visible, this should have happened very recently. Given that the linear size of the MSO in the centre of B $0818+214$ is a few kpc and assuming a lobe expansion rate of the order of $0.1 c$ (Scheuer 1995), it appears that the cessation of activity happened after a relatively short active period with a length of the order of $10^{5} \mathrm{yr}$. The duration of the active period may be even shorter because lobe expansion rates for DDRSs could equally well be even $0.3 c$ as shown by Schoenmakers et al. (2000b) for B1834+620. Therefore, the inner part of B $0818+214$, like other MSOs, could be intermittent on a timescale of $10^{4}-10^{5} \mathrm{yr}$ as originally theorised by Reynolds \& Begelman (1997). Recently, a short-timescale intermittency in young radio sources resulting from radiation-pressure-driven instabilities in accretion disks was modelled by Czerny et al. (2009) and studied on samples of GPS/CSS sources by Wu (2009a,b).

The assumption that the MSO in the centre of B $0818+214$ is in the coasting phase leads to the conclusion that the behaviour of the active nucleus in B $0818+214$ fits two different evolutionary tracks. On the one hand, its outer lobes resulted from a long phase of activity interrupted due to e.g. ionisation instability operating on longer timescales (Hatziminaoglou et al. 2001; Janiuk et al. 2004, and references therein). On the other hand, in the most recent activity cycle, the central source is intermittent due to radiation-pressure instability operating on much shorter timescales (Czerny et al. 2009). Hence, it appears that two accretion disk instability mechanisms, ionisation and radiationpressure driven, could be at work in the same galaxy and the outburst resulting from the short-timescale instability may occur during the quiescent phase of the long-timescale instability. A direct consequence of this scenario is that B $0818+214$ in its present form would not evolve to a standard DDRS.

\section{Concluding remarks}

Recurrent activity in galaxies is likely to be much more ubiquitous than it appears and the re-triggering timescales can span several orders of magnitude. The main reason for why we do not see many such events in the form of a DDRS is that young jets often travel silently in the cocoon inflated during the previous active phase because of its low density and so they do not develop shocks/hotspots. On the other hand, the quiescent state of an active nucleus for a given cycle can be longer than the decay time of the outer lobes. This could explain the negative result of the search for relics carried out by Sirothia et al. (2009). Therefore, it looks as if some fine-tuning between the lifetime of the outer coasting structures and the time needed to refill the cocoon is 
required to make it possible for a standard DDRS phenomenon to take place and that fine-tuning often does not work.

Out of the wide spectrum of sizes of the inner doubles, only radio sources with either the largest or the smallest ones can be observed: the former are perceived as classical DDRSs, whereas the latter appear as CDTs and only high-resolution observations of their "cores" can reveal their true nature. For the latter group of objects, a sub-galactic size of the inner double is likely so that the radio source is embedded in the host galaxy. This circumstance makes the above-mentioned fine-tuning not necessary for them as the ISM is always dense enough for an FR II-like structure to develop and so whether the outer cocoon has already been refilled or not is irrelevant. CDTs are, therefore, a potential reservoir of numerous DDRSs with the caveat that not every CDT will turn out to be a DDRS. For example, Marecki (2004) presented a high-resolution observation of a CDT source J1708+0035 whose central component is not a compact double but a core-jet structure. Moreover, a conspicuous misalignment between the axes of the inner and the outer structures is present in that source which makes it belong to a different class of object. Further specimens of this class are shown and an interpretation is given in Paper I.

Acknowledgements. The European VLBI Network is a joint facility of European, Chinese, South African and other radio astronomy institutes funded by their national research councils. MERLIN is operated by the University of Manchester as a National Facility on behalf of the Particle Physics \& Astronomy Research Council (PPARC). A.M. acknowledges the receipt of a travel grant funded by FP6 RadioNet as a part of the Trans-National Access (TNA) programmes.

\section{References}

Becker, R. H., White, R. L., \& Edwards, A. L. 1991, ApJS, 75, 1

Best, P. N. 2007, New Astron. Rev., 51, 168

Brocksopp, C., Kaiser, C. R., Schoenmakers, A. P., \& de Bruyn, A. G. 2007, MNRAS, 382, 1019

Czerny, B., Siemiginowska, A., Janiuk, A., Nikiel-Wroczyński, B., \& Stawarz, Ł. 2009, ApJ, 698, 840
Fanaroff, B. L., \& Riley, J. M. 1974, MNRAS, 167, 31P

Fanti, C., Fanti, R., Dallacasa, D., et al. 1995, A\&A, 302, 317

Hatziminaoglou, E., Siemiginowska, A., \& Elvis, M. 2001, ApJ, 547, 90

Holt, J., Tadhunter, C. N., \& Morganti, R. 2006, Astron. Nachr., 327, 147

Jamrozy, M., Saikia, D. J., \& Konar, C. 2009, MNRAS, 399, L141

Janiuk, A., Czerny, B., Siemiginowska, A., \& Szczerba, R. 2004, ApJ, 602, 595

Jeyakumar, S., Wiita, P. J., Saikia, D. J., \& Hooda, J. S. 2005, A\&A, 432, 823

Kaiser, C. R., Schoenmakers, A. P., \& Röttgering, H. J. A. 2000, MNRAS, 315, 381 (KSR)

Komissarov, S. S., \& Gubanov, A. G. 1994, A\&A, 285, 27

Konar, C., Saikia, D. J., Ishwara-Chandra, C. H., \& Kulkarni, V. K. 2004, MNRAS, 355, 845

Labiano, A. 2008, A\&A, 488, L59

Labiano, A., O'Dea, C. P., Gelderman, R., de Vries, W. H., \& Axon, D. J. 2005, A\&A, 436, 493

Labiano, A., Vermeulen, R. C., Barthel, P. D., et al. 2006, A\&A, 447, 481

Lara, L., Márquez, I., Cotton, W. D., et al. 1999, A\&A, 348, 699

Marecki, A., Barthel, P. D., Polatidis, A., \& Owsianik, I. 2003, PASA, 20, 16

Marecki, A. 2004, Proceedings of the 7th European VLBI Network Symposium, 12-15 October 2004, Toledo, ed. R. Bachiller et al., 117

Marecki, A., Thomasson, P., Mack, K.-H., \& Kunert-Bajraszewska, M. 2006, A\&A, 448, 479 (Paper I)

Morganti, R., Killeen, N. E. B., \& Tadhunter, C. N. 1993, MNRAS, 263, 1023

Reynolds, C. S., \& Begelman, M. C. 1997, ApJ, 487, L135

Richstone, D., Ajhar, E. A., Bender, R., et al. 1998, Nature, 395, A14

Saikia, D. J., Konar, C., \& Kulkarni, V. K. 2006, MNRAS, 366, 1391

Saripalli, L., Subrahmanyan, R., \& Udaya Shankar, N. 2002, ApJ, 565, 256

Saripalli, L., Subrahmanyan, R., \& Udaya Shankar, N. 2003, ApJ, 590, 181

Schilizzi, R. T., Tian, W. W., Conway, J. E., et al. 2001, A\&A, 368, 398

Schoenmakers, A. P., Bruyn, A. G., Röttgering, H. J. A., van der Laan, H., \& Kaiser, C. R. 2000a, MNRAS, 315, 371

Schoenmakers, A. P., Bruyn, A. G., Röttgering, H. J. A., \& van der Laan, H. 2000b, MNRAS, 315, 395

Scheuer, P. A. G. 1995, MNRAS, 277, 331

Sirothia, S. K., Saikia, D. J., Ishwara-Chandra, C. H., \& Kantharia, N. G. 2009, MNRAS, 392, 1403

Slee, O. B., Roy, A. L., Murgia, M., Andernach, H., \& Ehle, M. 2001, AJ, 122, 1172

Spergel, D. N., Verde, L., Peiris, H. V., et al. 2003, ApJS, 148, 175

White, R. L., Becker, R. H., Helfand, D. J., \& Gregg, M. D. 1997, ApJ, 475, 479 Wu, Q. 2009a, MNRAS, 398, 1365

Wu, Q. 2009b, ApJ, 701, L95 